\title{
Novel Dual Band Microstrip Circular Patch Antennas Loaded with ENG and MNG Metamaterials
}

\author{
Md. Ababil Hossain, ${ }^{1}$ Md. Saimoom Ferdous, ${ }^{2}$ \\ Shah Mahmud Hasan Chowdhury, ${ }^{1}$ and Md. Abdul Matin ${ }^{1}$ \\ ${ }^{1}$ Department of Electrical and Electronic Engineering, BUET, Dhaka 1000, Bangladesh \\ ${ }^{2}$ Department of ECE, University of British Columbia, Okanagan, BC, Canada
}

Correspondence should be addressed to Md. Ababil Hossain; ababilh@gmail.com

Received 13 September 2013; Accepted 20 November 2013; Published 11 February 2014

Academic Editor: Guo Qing Luo

Copyright (c) $2014 \mathrm{Md}$. Ababil Hossain et al. This is an open access article distributed under the Creative Commons Attribution License, which permits unrestricted use, distribution, and reproduction in any medium, provided the original work is properly cited.

Novel design of a dual band microstrip circular patch antenna loaded with ENG ( $\varepsilon$ negative) metamaterial has been shown in the first section. Using ENG metamaterial instead of the conventional dielectric, unconventional $\operatorname{TM}_{\delta 10}(1<\delta<2)$ mode was produced to yield a dual band performance. Optimized parameters such as permittivity $\left(\varepsilon_{1}\right)$ and filling ratio $(\eta)$ of metamaterials were selected with the aid of a MATLAB based parameter optimization algorithm, developed for all these sort of patch antennas. In the second section, a dual band circular patch antenna loaded with MNG ( $\mu$ negative) metamaterial has been reported. An unconventional modified $\mathrm{TM}_{\delta 10}(0<\delta<1)$ mode has been produced along with conventional $\mathrm{TM}_{110}$ mode due to using MNG metamaterial. Here also the optimum values of permeability $\left(\mu_{1}\right)$ and filling ratio $(\eta)$ for these sorts of patch antennas have been calculated from a MATLAB based parameter optimization algorithm. Both the proposed antennas provide good and resonance and satisfactory radiation performances (directivity, radiation efficiency, and gain) with a dual band performance.

\section{Introduction}

Satellite communications, wireless communications, surveillance, weather, radar, and so forth require highly directive and multiband antennas. In fact, in recent times, highly directive multiband antennas have become a prime concern in satellite communication.

The use of multiband antenna has brought about revolutionary change and dynamism in the trends of communication over the last few decades. However, antenna size miniaturization is also an important factor as far as antenna size is concerned. Therefore, a unique radiating element such as patch antenna which incorporates multiband performance with size miniaturization can further open up a new horizon in telecommunication sector. In [1], a generalized MATLAB based parameter optimization algorithm for designing circular patch antenna loaded with metamaterials has been developed. On the basis of that design algorithm, in this paper we have presented the design model for dual band antennas loaded with both ENG and MNG metamaterials without the use of any symmetrical slotting which was done in $[1,2]$.

Wong and Hsieh [2] constructed dual band circular patch antenna by using conventional dielectrics as substrate and by using symmetrical slots where conventional $\mathrm{TM}_{210}$ mode was modified to yield dual band performance. In that case the first order mode $\left(\mathrm{TM}_{110}\right.$ mode frequency) and second order mode $\left(\mathrm{TM}_{210}\right.$ mode frequency) were determined by the antenna geometry structure (i.e., radius of the patch). Once the one mode has been selected, the other mode frequency automatically becomes fixed. In that case the designer could only tune any one mode frequency according to his will; he had no control over choosing the other mode frequency as the other mode frequency is determined by antenna radius. In that particular case the degree of freedom was 
only one, that is, radius of the patch. So with one degree of freedom designers could choose only one resonant frequency independently, but not both at the same time.

In the first section of this paper, a novel design of dual band ENG metamaterial loaded circular patch antenna has been shown. Its main feature is in the flexibility of choosing both resonant frequencies according to user's will and apart from conventional $\mathrm{TM}_{110}$ mode newly produced unconventional $\mathrm{TM}_{\delta 10}(1<\delta<2)$ mode provides high directivity. Our designed antenna has two degrees of freedom (radius of the patch and filling ratio of the metamaterial). In our case, the first band, that is, first order mode, is determined by the patch geometry only. Using ENG metamaterial instead of conventional dielectrics causes resonance in between first and second order mode frequency. For this reason, the biggest advantage here is that the designer can tune the second band almost anywhere in between conventional first and second order mode by just changing the filling ratio of the metamaterial. That is why this design provides a great deal of flexibility in choosing resonant frequency in comparison to other dual band circular patch antennas reported so far.

Alù et al. proposed design method to obtain electrically small rectangular patch antennas using DPS (double positive) ENG metamaterial juxtaposed layer [3]. But such rectangular patches give broadside null radiation pattern at subwavelength regime as illustrated in Figure 2 of [4]. Finally, it was predicted that all these electrically small rectangular antennas loaded with metamaterial can only be good resonators but may not be good radiators. But if the shape is circular $[4,5]$ or elliptical [6], theoretically it is possible to achieve electrically small size without deterioration of radiation performance. With this concept we developed a proper algorithm in [1], where achieving additional unconventional mode in metamaterial loaded circular patch antenna has been possible. By using the same algorithm, here a dual band ENG metamaterial loaded patch antenna has been designed. Highly directive performance and flexibility in tuning frequency are the two prime criteria of this dual band patch antenna.

In the second section of this paper, by using MNG ( $\mu$ negative) metamaterial in the inner core as substrate instead of natural dielectric in circular microstrip patch antenna, dual band performance has been achieved with a reduced size antenna. Apart from conventional $\mathrm{TM}_{110}$ mode, here unconventional $\operatorname{TM}_{\delta 10}(0<\delta<1)$ mode has been produced. Applying our proposed design algorithm [1], we can achieve broadside radiation at $\mathrm{TM}_{\delta 10}(0<\delta<1)$ mode and hence achieve a dual band performance with a reduced size antenna. In this sort of metamaterial loaded patch antennas, the resonant frequency can be tuned according to designer's will. But it has been shown that electrically small size patch antenna having metamaterial loading is not practicable without degradation of radiation performance $[7,8]$. In fact, the gain performances of such electrically small antennas degrade rapidly due to the size reduction. However, nearly $35 \%$ size reduction may be possible where gain remains above $0 \mathrm{~dB}[8]$. In our proposed designed antenna based on our developed algorithm, we have obtained around 30\% size reduction with a gain of $2.2 \mathrm{~dB}$. Flexibility in tuning resonant frequency along with size miniaturization has certainly made this dual band patch antenna unique from other dual band antennas reported in current literature.

MNG metamaterial can be fabricated in the laboratory at microwave frequencies by using Split Ring Resonators (SRR) and helices, whereas thin wired structure placed at a periodic distance works as negative Epsilon material when the distance between consecutive wires becomes comparable to the wavelength of the propagating electromagnetic wave. Over the years, a lot of research has been carried out in ENG loaded patch antenna, but ironically the practical implementation of ENG metamaterial at microwave regime using Lorentz model is a tough task [9]. However, recent advancement of ENG medium applications in microwave regime urges its practical implementation. Although the practical application of ENG medium using Lorentz model in microwave regime is really a tough one, the directivity and gain performance of such antennas are attractive with conventional size.

\section{Mathematical Modeling of Metamaterial Loaded Circular Microstrip Patch Antenna}

The basic magnetization vector equation for a circular patch antenna can be written as

$$
\begin{aligned}
A_{z}^{n}(\rho, \phi, z)= & {\left[A_{m n} J_{m}\left(k_{\rho n} \rho\right)+B_{m n} Y_{m}\left(k_{\rho n} \rho\right)\right] } \\
& \times\left[C_{n} \cos (m \phi)+D_{n} \sin (m \phi)\right] \\
& \times\left[C_{3} \cos \left(k_{z n} z\right)+D_{3} \sin \left(k_{z n} z\right)\right] .
\end{aligned}
$$

But radial component of the electric field can be derived as

$$
E_{\rho}^{n}=-j \frac{1}{\omega \varepsilon_{n} \mu_{n}} \frac{\partial^{2}}{\partial \rho \partial z}\left(A_{z}^{n}\right) .
$$

Here, $n=1$ for region 1 (metamaterial region) and $n=2$ for region 2 (conventional dielectric region).

From boundary conditions (perfect electric wall assumption of cavity model),

$$
\begin{aligned}
& \text { at } z=h, E_{\rho}=0 \text {, we get } D_{3}=0, \\
& \text { and at } z=0, E_{\rho}=0 \text {, then we get } C_{3}=1 .
\end{aligned}
$$

Therefore,

$$
\begin{aligned}
A_{z}^{n}(\rho, \phi, z)= & {\left[A_{m n} J_{m}\left(k_{\rho n} \rho\right)+B_{m n} Y_{m}\left(k_{\rho n} \rho\right)\right] } \\
& \times\left[C_{n} \cos (m \phi)+D_{n} \sin (m \phi)\right] \\
& \times \cos \left(k_{z n} z\right), \\
E_{\rho}^{n}(\rho, \phi, z)= & -j \frac{k_{\rho n} k_{z n}}{\omega \varepsilon_{n} \mu_{n}} \\
& \times\left[A_{m n} J_{m}^{\prime}\left(k_{\rho n} \rho\right)+B_{m n} Y_{m}^{\prime}\left(k_{\rho n} \rho\right)\right] \\
& \times\left[C_{n} \cos (m \phi)+D_{n} \sin (m \phi)\right] \sin \left(k_{z n} z\right) .
\end{aligned}
$$


Generalized equation for the phi component of the electric field is as follows:

$$
E_{\phi}^{n}=-j \frac{1}{\omega \varepsilon_{n} \mu_{n}} \frac{1}{\rho} \frac{\partial^{2}}{\partial \phi \partial z}\left(A_{z}^{n}\right) .
$$

By calculation from the equation of $A_{z}^{n}$ we get

$$
\begin{aligned}
E_{\phi}^{n}(\rho, \phi, z)= & j \frac{k_{z n} m}{\rho \omega \varepsilon_{n} \mu_{n}} \\
& \times\left[A_{m n} J_{m}\left(k_{\rho n} \rho\right)+B_{m n} Y_{m}\left(k_{\rho n} \rho\right)\right] \\
& \times\left[-C_{n} \sin (m \phi)+D_{n} \cos (m \phi)\right] \sin \left(k_{z n} z\right) .
\end{aligned}
$$

Finally, $z$-component of electric field becomes

$$
E_{z}^{n}=-\frac{j}{\omega \varepsilon_{n} \mu_{n}}\left(\frac{\partial^{2}}{\partial z^{2}}+k_{n}^{2}\right) A_{z}^{n}
$$

Again, calculation from the equation of $A_{z}^{n}$ yields

$$
\begin{aligned}
E_{z}^{n}(\rho, \phi, z)= & -j \frac{k_{\rho n}^{2}}{\omega \varepsilon_{n} \mu_{n}}\left[A_{m n} J_{m}\left(k_{\rho n} \rho\right)+B_{m n} Y_{m}\left(k_{\rho n} \rho\right)\right] \\
& \times\left[C_{n} \cos (m \phi)+D_{n} \sin (m \phi)\right] \cos \left(k_{z n} z\right) .
\end{aligned}
$$

For the magnetic fields component of the cavity, $z$-component of the magnetic field is 0 everywhere.

So,

$$
H_{z}^{n}=0 .
$$

Again, radial component of the magnetic field is formulated as

$$
\begin{aligned}
H_{\rho}^{n}= & \frac{1}{\rho \mu_{n}} \frac{\partial A_{z}^{n}}{\partial \phi} \\
= & \frac{m}{\rho \mu_{n}}\left[A_{m n} J_{m}\left(k_{\rho n} \rho\right)+B_{m n} Y_{m}\left(k_{\rho n} \rho\right)\right] \\
& \times\left[-C_{n} \sin (m \phi)+D_{n} \cos (m \phi)\right] \cos \left(k_{z n} z\right) .
\end{aligned}
$$

Finally,

$$
H_{\phi}^{n}=-\frac{1}{\mu_{n}} \frac{\partial A_{z}^{n}}{\partial \rho}
$$

which gives

$$
\begin{aligned}
H_{\phi}^{n}= & -\frac{k_{\rho n}}{\mu_{n}}\left[A_{m n} J_{m}^{\prime}\left(k_{\rho n} \rho\right)+B_{m n} Y_{m}^{\prime}\left(k_{\rho n} \rho\right)\right] \\
& \times\left[C_{n} \cos (m \phi)+D_{n} \sin (m \phi)\right] \cos \left(k_{z n} z\right) .
\end{aligned}
$$

For all the above equations, $J_{m}$ and $J_{m}^{\prime}$ are Bessel functions of first kind and its derivative denoted by prime order of $m . Y_{m}$ and $Y_{m}^{\prime}$ are Bessel functions of second kind and its derivative denoted by prime order of $m$.

Now applying boundary condition at the interface, that is, at $\rho=b, E_{Z}^{1}=E_{Z}^{2}$ we get from electric field equations,

$$
\begin{gathered}
{\left[\frac{k_{\rho 1}^{2}}{\mu_{1} \epsilon_{1}} J_{m}\left(k_{\rho 1} b\right)\right] A_{m 1}-\left[\frac{k_{\rho 2}^{2}}{\mu_{2} \epsilon_{2}} J_{m}\left(k_{\rho 2} b\right)\right] A_{m 2}} \\
-\left[\frac{k_{\rho 2}^{2}}{\mu_{2} \epsilon_{2}} Y_{m}\left(k_{\rho 2} b\right)\right] B_{m 2}=0 .
\end{gathered}
$$

Again, at $\rho=b, H_{\phi}^{1}=H_{\phi}^{2}$, we get from magnetic field equations that

$$
\begin{gathered}
{\left[\frac{k_{\rho 1}}{\mu_{1}} J_{m}^{\prime}\left(k_{\rho 1} b\right)\right] A_{m 1}-\left[\frac{k_{\rho 2}}{\mu_{2}} J_{m}^{\prime}\left(k_{\rho 2} b\right)\right] A_{m 2}} \\
-\frac{k_{\rho 2}}{\mu_{2}} Y_{m}^{\prime}\left(k_{\rho 2} b\right) B_{m 2}=0 .
\end{gathered}
$$

Finally, at $\rho=a, H_{\phi}^{2}=0$ and then we get similarly

$$
\left[\frac{k_{\rho 2}}{\mu_{2}} J_{m}^{\prime}\left(k_{\rho 2} a\right)\right] A_{m 2}+\left[\frac{k_{\rho 2}}{\mu_{2}} Y_{m}^{\prime}\left(k_{\rho 2} a\right)\right] B_{m 2}=0 .
$$

From the previous three equations, that is, (13), (14), and (15), we see that the right side is zero. So, in order to have solutions, the determinant formed by the corresponding matrix must be equal to 0 :

$$
\left|\begin{array}{ccc}
\frac{k_{\rho 1}^{2}}{\mu_{1} \epsilon_{1}} J_{m}\left(k_{\rho 1} b\right) & -\frac{k_{\rho 2}^{2}}{\mu_{2} \epsilon_{2}} J_{m}\left(k_{\rho 2} b\right) & -\frac{k_{\rho 2}^{2}}{\mu_{2} \epsilon_{2}} Y_{m}\left(k_{\rho 2} b\right) \\
\frac{k_{\rho 1}}{\mu_{1}} J_{m}^{\prime}\left(k_{\rho 1} b\right) & -\frac{k_{\rho 2}}{\mu_{2}} J_{m}^{\prime}\left(k_{\rho 2} b\right) & -\frac{k_{\rho 2}}{\mu_{2}} Y_{m}^{\prime}\left(k_{\rho 2} b\right) \\
0 & \frac{k_{\rho 2}}{\mu_{2}} J_{m}^{\prime}\left(k_{\rho 2} a\right) & \frac{k_{\rho 2}}{\mu_{2}} Y_{m}^{\prime}\left(k_{\rho 2} a\right)
\end{array}\right|=0 .
$$

Solving the above determinant we get

$$
\begin{aligned}
& \frac{k_{\rho 1}}{\epsilon_{1}} \frac{J_{m}\left(k_{\rho 1} b\right)}{J_{m}^{\prime}\left(k_{\rho 1} b\right)} \\
& =\frac{k_{\rho 2}}{\epsilon_{2}}\left[\frac{J_{m}\left(k_{\rho 2} a\right) Y_{m}^{\prime}\left(k_{\rho 2} a\right)-J_{m}^{\prime}\left(k_{\rho 2} a\right) Y_{m}\left(k_{\rho 2} b\right)}{J_{m}^{\prime}\left(k_{\rho 2} b\right) Y_{m}^{\prime}\left(k_{\rho 2} a\right)-J_{m}^{\prime}\left(k_{\rho 2} a\right) Y_{m}^{\prime}\left(k_{\rho 2} b\right)}\right] .
\end{aligned}
$$

Now for ENG metamaterial,

$$
k_{\rho 1} \approx k_{1}=j \omega \sqrt{\mu_{1}\left|\epsilon_{1}\right|} .
$$

And for MNG metamaterial,

$$
k_{\rho 1} \approx k_{1}=j \omega \sqrt{\epsilon_{1}\left|\mu_{1}\right|} .
$$




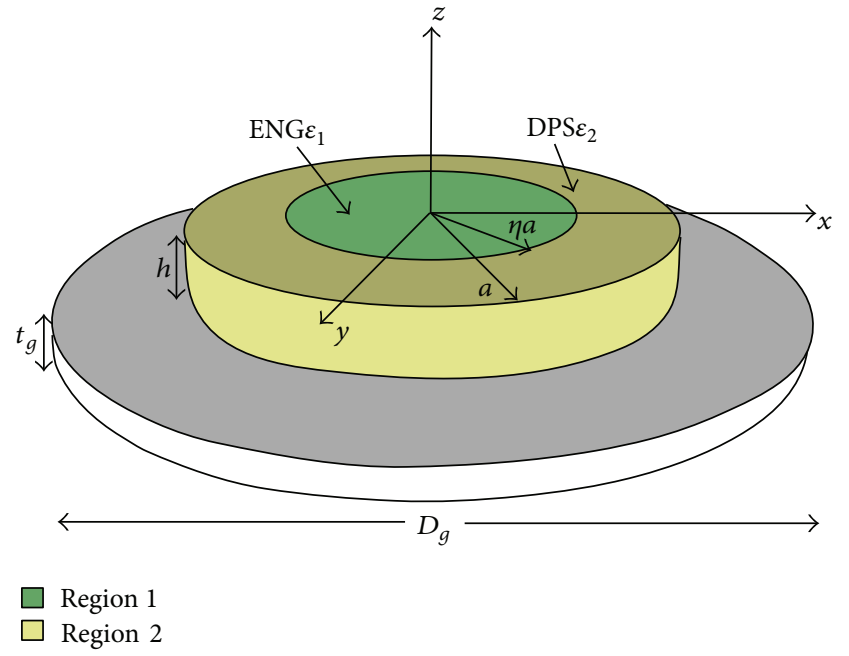

FIGURE 1: Geometry of a circular microstrip patch antenna partially loaded with metamaterial (ENG) substrate and ground plane with parameters $\eta a=(.56)(20 \mathrm{~mm})=11.2 \mathrm{~mm}, a=20 \mathrm{~mm}, h=$ $4.3 \mathrm{~mm}, t_{g}=2 \mathrm{~mm}, D_{g}=40 \mathrm{~mm}, \varepsilon_{2}=1.33, \varepsilon_{1}=-1.9$ (at $4.2686 \mathrm{GHz}$ ), $\mu_{2}=1, \mu_{1}=1$, and feed position from the centre $f_{p}=15 \mathrm{~mm}$.

TABLE 1: Selected permittivity and filling ratio for ENG-metamaterial loading in antenna.

\begin{tabular}{lcc}
\hline $\begin{array}{l}\text { Chosen resonant } \\
\text { frequency, } f_{r}(\mathrm{GHz})\end{array}$ & $\varepsilon_{1}\left(\right.$ at $\left.f_{r}\right)$ & Filling ratio $(\eta)$ \\
\hline 4.2686 & -1.90 & 0.56 \\
\hline
\end{tabular}

Putting the above relation, the dispersion relation for ENG metamaterial loaded patch antenna becomes

$$
\begin{aligned}
& 2 \sqrt{\frac{\mu_{1} \epsilon_{2}}{\mu_{2}\left|\epsilon_{1}\right|}} \frac{I_{m}\left(\left|k_{\rho 1} b\right|\right)}{I_{m-1}\left(\left|k_{\rho 1}\right| b\right)+I_{m+1}\left(\left|k_{\rho 1}\right| b\right)} \\
& =\frac{J_{m}\left(k_{\rho 1} b\right) Y_{m}^{\prime}\left(k_{\rho 1} a\right)-J_{m}^{\prime}\left(k_{\rho 1} a\right) Y_{m}^{\prime}\left(k_{\rho 1} b\right)}{J_{m}^{\prime}\left(k_{\rho 2} b\right) Y_{m}^{\prime}\left(k_{\rho 2} a\right)-J_{m}^{\prime}\left(k_{\rho 2} a\right) Y_{m}^{\prime}\left(k_{\rho 2} b\right)} .
\end{aligned}
$$

And for MNG metamaterial, the dispersion relation is

$$
\begin{aligned}
& -2 \sqrt{\frac{\left|\mu_{1}\right| \epsilon_{2}}{\mu_{2} \epsilon_{1}} \frac{I_{m}\left(\left|k_{\rho 1} b\right|\right)}{I_{m-1}\left(\left|k_{\rho 1}\right| b\right)+I_{m+1}\left(\left|k_{\rho 1}\right| b\right)}} \\
& =\frac{J_{m}\left(k_{\rho 1} b\right) Y_{m}^{\prime}\left(k_{\rho 1} a\right)-J_{m}^{\prime}\left(k_{\rho 1} a\right) Y_{m}^{\prime}\left(k_{\rho 1} b\right)}{J_{m}^{\prime}\left(k_{\rho 2} b\right) Y_{m}^{\prime}\left(k_{\rho 2} a\right)-J_{m}^{\prime}\left(k_{\rho 2} a\right) Y_{m}^{\prime}\left(k_{\rho 2} b\right)} .
\end{aligned}
$$

By using the above two dispersion relations, a MATLAB based parameter optimization algorithm has been developed in [1]. In that algorithm, the optimized value of metamaterial parameter such as filling ratio, permittivity, and permeability has been calculated for metamaterial loaded circular patch antennas.

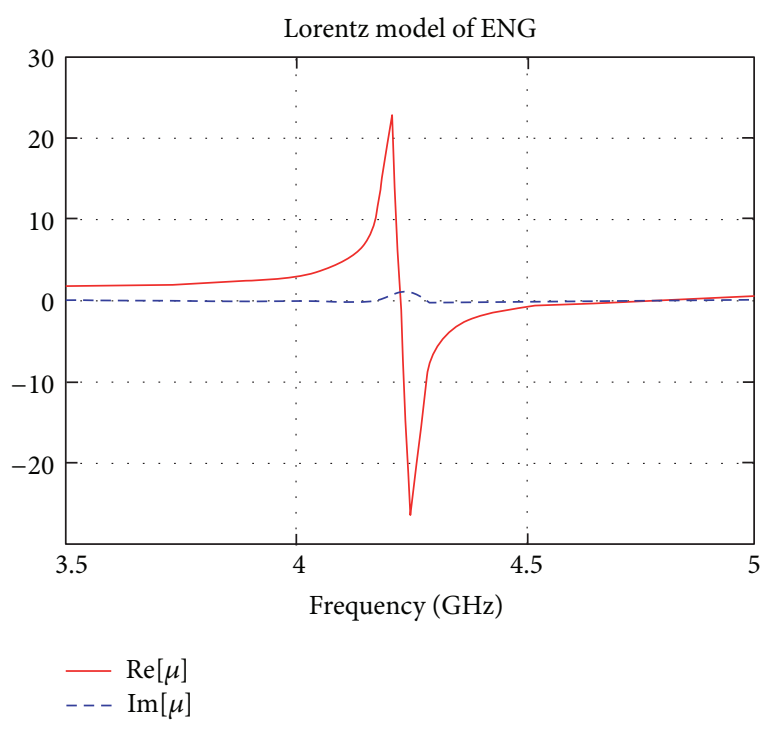

FIGURE 2: Lorentz dispersive model of ENG for proposed antenna.

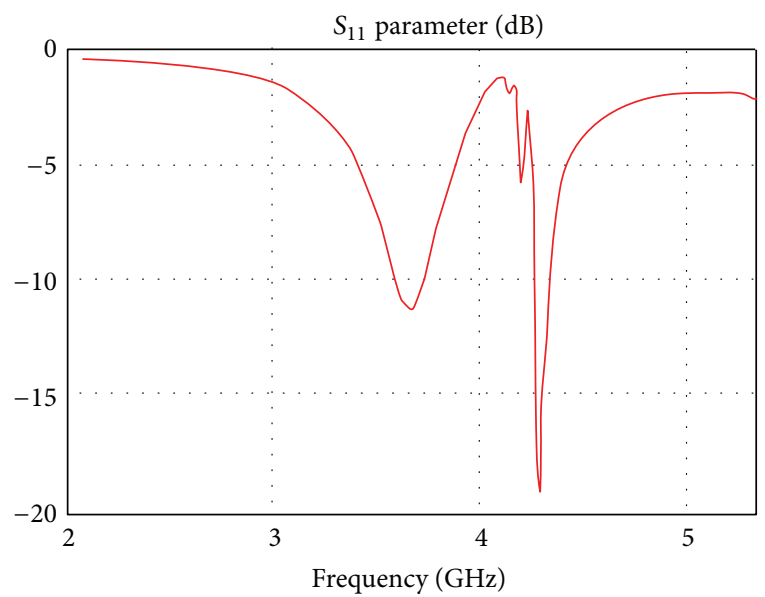

FIGURE 3: $S_{11}$ parameter for $20 \mathrm{~mm}$ circular patch antenna using ENG as core material.

\section{ENG Metamaterial Loaded Dual Band Circular Patch Antenna}

3.1. Antenna Design Structure and Specifications. In circular microstrip patch antenna, by using ENG as a substrate instead of regular dielectric we can achieve dual band performance. Apart from conventional $\mathrm{TM}_{110}$ mode unconventional $\mathrm{TM}_{\delta 10}$ $(1<\delta<2)$ mode can be modified.

In designing our proposed antenna, geometry of a circular patch with metamaterial block as shown in Figure 1 has been used. With proper choice of filling ratio $(\eta)$, ENG metamaterial $\left(\varepsilon_{1}\right)$ is loaded concentrically with regular dielectric $\left(\varepsilon_{2}\right)$. In Figure 1 ENG metamaterial is denoted by region 1 (green) and regular dielectric is denoted by region 2 (yellow). The antenna consists of a metallic patch of thickness $\left(t_{p}=1 \mathrm{~mm}\right)$ and radius $(a=20 \mathrm{~mm})$ at the top. A metallic plate placed at the bottom with radius $(2 \times a=40 \mathrm{~mm})$ and 


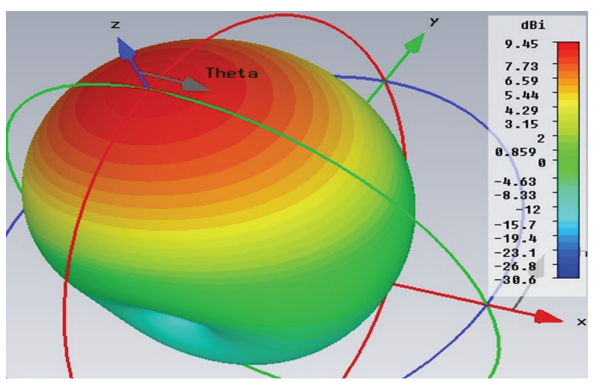

(a)

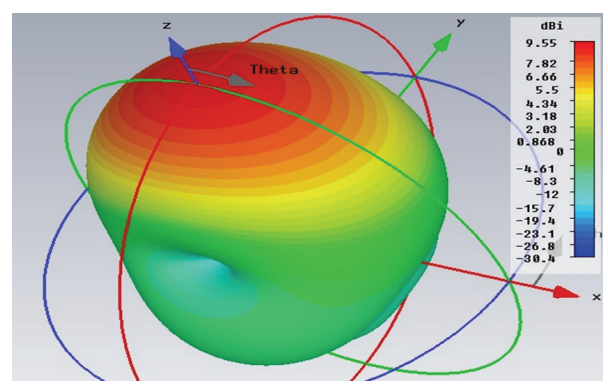

(b)

Figure 4: 3D view of (a) conventional $\mathrm{TM}_{110}$ mode at $3.6665 \mathrm{GHz}$ and (b) unconventional $\mathrm{TM}_{\delta 10}(1<\delta<2)$ mode's radiation pattern at $4.2686 \mathrm{GHz}$.

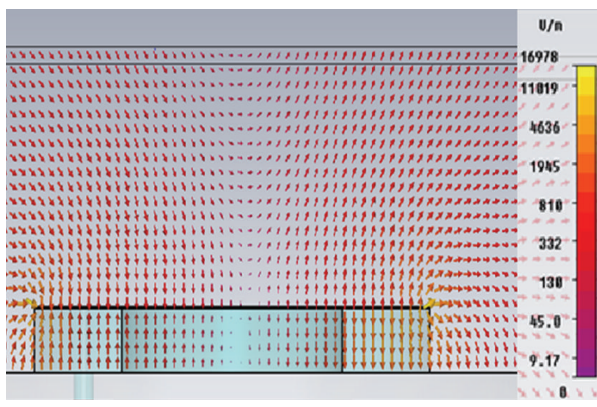

(a)

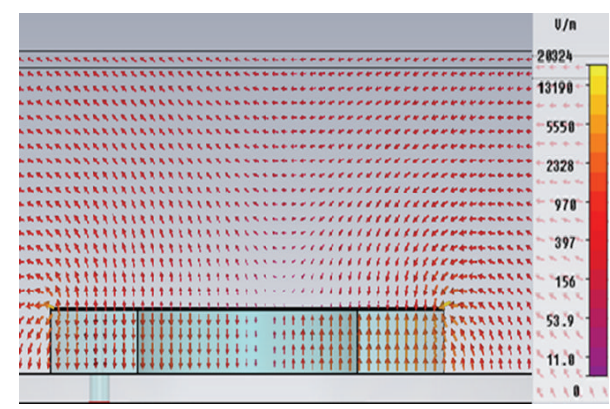

(b)

Figure 5: Electric field distribution on the $y=0$ plane at (a) $f_{110}=3.6665 \mathrm{GHz}$ and (b) $f_{\delta 10}(1<\delta<2)=4.2686 \mathrm{GHz}$.

thickness $\left(t_{g}=2 \mathrm{~mm}\right)$ acts as ground. This chosen optimum radius and thickness of the ground plate causes maximum reflection from the ground that enhances the directivity. Conductivity of the metal is selected as $0.58 \mathrm{Meg} \sigma$. As a feed we have used coaxial cable designed to have $Z_{p}=50 \Omega$ characteristic impedance with inner radius of coaxial cable $\left(r_{\text {in }}=0.4 \mathrm{~mm}\right)$ and outer radius of coaxial cable $\left(r_{\text {out }}=\right.$ $1.0456 \mathrm{~mm}$ ) and its position is set at $f_{p}=15 \mathrm{~mm}$ from the center for achieving good matching properties over operating frequency range. The underneath substrate is filled with concentric dielectrics with ENG metamaterial as the inner core and DPS material as outer core. Dielectric substrate height is $h=4.3 \mathrm{~mm}$. Of all the dielectric parameters, $\varepsilon_{2}=$ $1.3 \varepsilon_{0}, \mu_{2}=1.0 \mu_{0}$, and $\mu_{1}=1.0 \mu_{0}$ are chosen as the optimum values for these parameters. However, the rest two controlling parameters, that is, ENG metamaterial's permittivity $\left(\varepsilon_{1}\right)$ and filling ratio $(\eta)$ have been calculated by a MATLAB based parameter optimization algorithm [1] and with the aid of dispersive equation (20), developed for ENG metamaterial. In Table 1. those optimum parameters are shown at our desired resonant frequency of $4.26 \mathrm{GHz}$.

Actually, SNG or DNG metamaterials are inherently dispersive and lossy $[10,11]$. So, without using dispersive lossy model the simulated results cannot give proper realistic results. Here we have used Lorentz model for ENG metamaterial dispersive relation:

$$
\varepsilon(\omega)=\varepsilon_{\infty}+\frac{\left(\varepsilon_{s}-\varepsilon_{\infty}\right) \omega_{0}^{2}}{\omega_{0}^{2}-\omega^{2}-j \omega \delta},
$$

where $\varepsilon_{\infty}=1.0 \varepsilon_{0}, \varepsilon_{s}=1.23 \varepsilon_{0}, \omega_{0}=26.6 \mathrm{Grad} / \mathrm{sec}, f_{r}=$ $4.26 \mathrm{GHz}$, and damping frequency $\delta=1 \mathrm{MHz}$.

Plugging these parameters into (22) dispersive relation of our desired ENG material should be as in Figure 2.

Using all the above stated material and geometric parameters, CST microwave studio [12] simulation gives the following results: $S$-parameter, 3D radiation patterns, and electric and current distribution (Figures 3-6).

3.2. Resonance and Radiation Characteristics of the Antenna. From the $S_{11}$ parameter curve of we see that corresponding return losses at $3.66 \mathrm{GHz}$ and $4.2686 \mathrm{GHz}$ frequencies fall well below $-10 \mathrm{~dB}$ that ensures satisfactory resonance for the antenna structure. But satisfactory resonance does not always guarantee good radiation. So in order to ensure good radiation and thus call it a good radiator, we need to look into its radiation patterns at those particular resonant frequencies also. The radiation patterns obtained at the resonant frequencies indicated at the $S_{11}$ parameter curve of Figure 3 are shown in Figure 4.

From the 3D radiation patterns of the ENG loaded patch antenna (see Figure 4), we see that conventional $\mathrm{TM}_{110}$ mode as well as the modified $\mathrm{TM}_{\delta 10}$ mode $(1<\delta<2)$ shows satisfactory $z$-directed radiation (Figures $4(\mathrm{a})$ and $4(\mathrm{~b})$ ). The conventional $\mathrm{TM}_{110}$ mode has the gain of $9.45 \mathrm{~dB}$, whereas the gain of the modified mode $\mathrm{TM}_{\delta 10}(1<\delta<2)$ at frequency $4.2686 \mathrm{GHz}$ is $9.55 \mathrm{~dB}$, which is even higher than the conventional mode. 


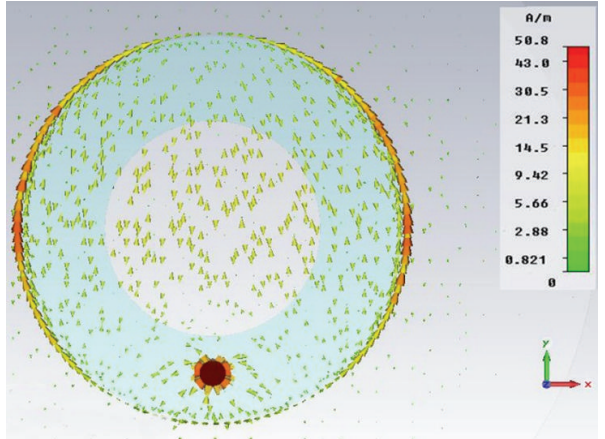

(a)

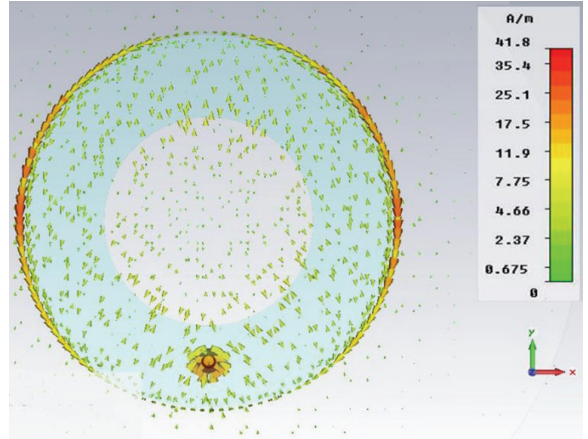

(b)

Figure 6: Current distribution on the patch at (a) $f_{110}=3.6665 \mathrm{GHz}$ and (b) $f_{\delta 10}(1<\delta<2)=4.2686 \mathrm{GHz}$.

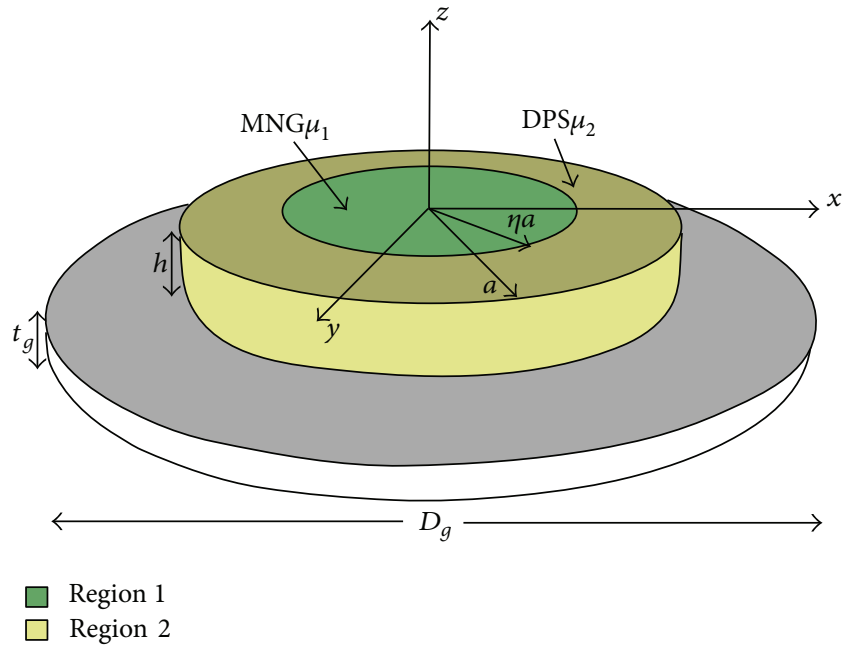

FIGURE 7: Geometry of a circular microstrip patch antenna partially loaded with metamaterial (MNG) substrate and ground plane with parameters: $\eta a=(.56 \mathrm{~mm})(20 \mathrm{~mm})=11.2 \mathrm{~mm}, a=20 \mathrm{~mm}, h=$ $5 \mathrm{~mm}, t_{g}=2 \mathrm{~mm}, D_{g}=40 \mathrm{~mm}, \varepsilon_{2}=1.33, \varepsilon_{1}=1.33, \mu_{2}=1$, $\mu_{1}=-2.83$ (at $2.56 \mathrm{GHz}$ ), and feed position $f_{p}=15 \mathrm{~mm}$.

3.3. Electric Field and Current Distribution. From the electric field distribution at the plane $y=0$, it is apparent that in case of conventional $\mathrm{TM}_{110}$ mode electric field flips its sign passing from one side to the other side of the patch, thus satisfying the condition for broadside radiation (Figure 5(a)). But in case of modified $\mathrm{TM}_{\delta 10}(1<\delta<2)$ mode due to using metamaterial, phase change of electric field at the ENG-DPS interface occurs in such a way that the electric field flips from one side to the other side of the patch, as a result of which the electric field distribution of $\mathrm{TM}_{\delta 10}(1<\delta<2)$ mode looks like that of $\mathrm{TM}_{110}$ mode, thus also satisfying the condition for broadside radiation (Figure 5(b)).

Microstrip circular patch antenna theory says that if the metallic patch induced current remains in the same phase passing from one side to the other side of the patch, that is, current distribution remains symmetric around $y$-axis, it must show broadside radiation. At $(x=0, y=0, z=0)$ point magnetic field distribution from the two opposite current

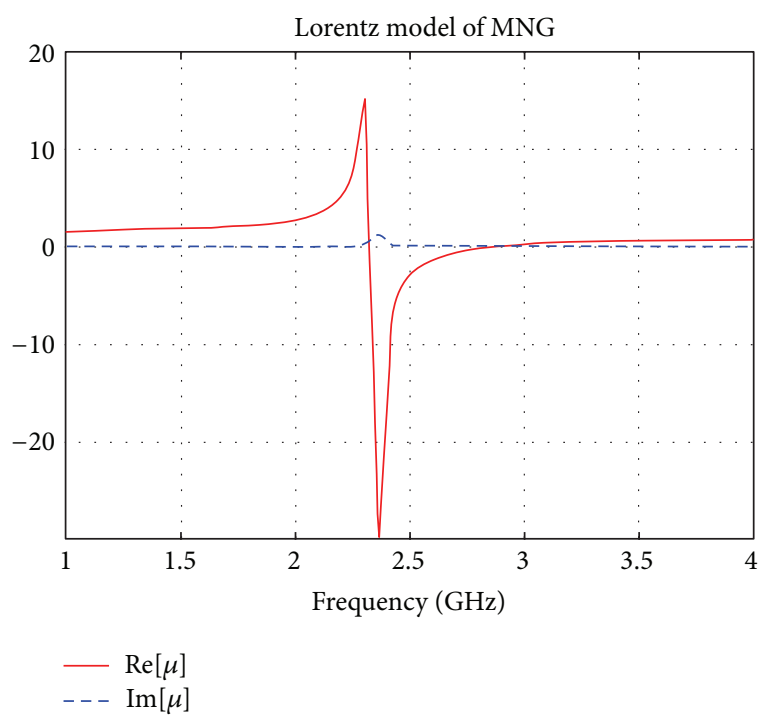

FIGURE 8: Lorentz dispersive model for proposed MNG loaded antenna.

elements reinforce in $z$ direction. That is why $\mathrm{TM}_{110}$ mode shows $z$-directed radiation (Figure 6(a)). But any other mode does not normally show this property. What metamaterial does is that, in our desired frequency, at interface of DPSENG, it causes phase change in such a way that the current distribution at that frequency resembles with $\mathrm{TM}_{110}$ mode's current distribution. Figure 6(b) shows current distribution of the patch for $\mathrm{TM}_{\delta 10}(1<\delta<2)$ mode that resembles with $\mathrm{TM}_{110}$ mode's pattern. It is noticeable that, despite the small dimension of the patch, the current distribution is closed in electrically small resonant loops and it resembles the current distribution of $\mathrm{TM}_{110}$ mode.

\section{MNG Metamaterial Loaded Dual Band Circular Patch Antenna}

4.1. Antenna Design Structure and Specifications. In designing our proposed antenna, geometry of a circular patch with metamaterial block as shown in Figure 7 has been used. It 


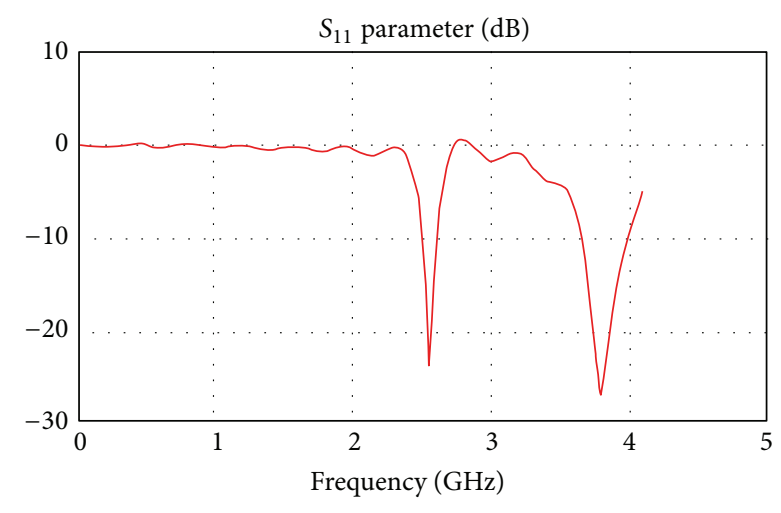

FIGURE 9: $S_{11}$-parameter for $20 \mathrm{~mm}$ radius circular patch antenna using MNG as core material.

is almost similar to the ENG loaded patch structure. It also consists of a metallic patch of thickness $\left(t_{p}=1 \mathrm{~mm}\right)$ and radius $(a=20 \mathrm{~mm})$ at the top. A metallic plate placed at the bottom with radius $(2 \times a=40 \mathrm{~mm})$ and thickness $\left(t_{g}=1 \mathrm{~mm}\right)$ acts as ground. This chosen optimum radius and thickness of the ground plate causes maximum reflection from the ground that enhances the directivity. The metal has a conductivity of $0.58 \mathrm{Meg} \sigma$. As a feed we have used coaxial cable designed to have $Z_{p}=50 \Omega$ characteristic impedance with inner radius of coaxial cable $\left(r_{\text {in }}=0.4 \mathrm{~mm}\right)$ and outer radius of coaxial cable $\left(r_{\text {out }}=1.0351 \mathrm{~mm}\right)$ and its position is set at $f_{p}=12 \mathrm{~mm}$ from the center for achieving good matching properties over operating frequency range. The underneath substrate is filled with concentric dielectrics with MNG metamaterial as the inner core and DPS material as outer core. Dielectric substrate height $(h=5 \mathrm{~mm})$ is spacious enough so as to allow realistic hosting of split ring resonator (SRR) to construct MNG material [13]. Of all the dielectric parameters, $\varepsilon_{2}=1.3 \varepsilon_{0}, \mu_{2}=1.0 \mu_{0}$, and $\varepsilon_{1}=$ $1.3 \varepsilon_{0}$ are chosen as the optimum values for these parameters. However, MNG metamaterial's permeability $\left(\mu_{1}\right)$ and filling ratio $(\eta)$ have been calculated by a MATLAB based parameter optimization algorithm [1] and with the aid of our derived dispersive equation (21) for MNG metamaterial. Calculated optimum permeability and filling ratio are shown in Table 2.

We have used Lorentz model for MNG metamaterial dispersive relation:

$$
\mu(\omega)=\mu_{\infty}+\frac{\left(\mu_{s}-\mu_{\infty}\right) \omega_{0}^{2}}{\omega_{0}^{2}-\omega^{2}-j \omega \delta}
$$

where $\mu_{\infty}=1.0 \mu_{0}, \mu_{s}=1.5 \mu_{0}$, and $\omega_{0}=14.76 \mathrm{Grad} / \mathrm{sec}$ causes resonance at $f_{r}=2.56 \mathrm{GHz}$ and damping frequency $(\delta=10 \mathrm{MHz})$. Putting these values in (3), we get the dispersion curve for MNG as shown in Figure 8.

4.2. Resonance and Radiation Characteristics. Using all the above stated material and geometric parameters, CST microwave studio [12] simulation gives the following results: $S$-parameter (Figure 9), 3D radiation patterns, and electric field distribution (Figures 10 and 11).
TABLE 2: Selected permeability $\left(\mu_{1}\right)$ and filling ratio $(\eta)$ for MNG loading.

\begin{tabular}{lcc}
\hline $\begin{array}{l}\text { Chosen resonant } \\
\text { frequency, } f_{r}(\mathrm{GHz})\end{array}$ & $\mu_{1}\left(\right.$ at $\left.f_{r}\right)$ & Filling ratio $(\eta)$ \\
\hline 2.56 & -2.83 & 0.35 \\
\hline
\end{tabular}

$S_{11}$ parameter curve, in Figure 9, shows that at frequencies $f_{\delta 10}(0<\delta<1)=2.56 \mathrm{GHz}, f_{110}=3.8 \mathrm{GHz}$ resonance occurs, which causes the corresponding return losses at these frequencies to fall well below $-10 \mathrm{~dB}$ that ensures satisfactory resonance. Again, from Figure 9, it is also evident that when antenna size is reduced, resonant frequency has to move away from the $\mathrm{TM}_{110}$ frequency which eventually causes to lose its $z$-directed radiation characteristics. So it is not always productive to reduce patch antenna size whimsically. But, in our study, we have found that around $70 \%$ size reduction provides us with nearly $-9 \mathrm{~dB}$ gain, whereas size reduction, that is,

$$
\begin{aligned}
& \frac{\left(f_{110}-f_{\delta 10}(0<\delta<1)\right)}{f_{110}} \times 100 \% \\
& =\frac{(3.66 \mathrm{GHz}-2.56 \mathrm{GHz})}{3.66 \mathrm{GHz}} \times 100 \% \approx 30 \%,
\end{aligned}
$$

provides us $2.2 \mathrm{~dB}$ gain which is quite satisfactory for practical antenna operation. This is the maximum allowable limit of size reduction with realizable gain performance that we have found in our study. In fact, antenna size reduction can be extended up to nearly $35 \%$ to get gain over $0 \mathrm{~dB}$.

From the 3D radiation patterns of MNG loaded antenna (see Figure 10) we see that conventional $\mathrm{TM}_{110}$ mode as well as the modified $\mathrm{TM}_{\delta 10}$ mode $(0<\delta<1)$ shows satisfactory $z$-directed radiation. The conventional mode $\mathrm{TM}_{110}$ has the gain $9.2 \mathrm{~dB}$ (Figure 10(b)) at frequency $3.8 \mathrm{GHz}$, whereas the gain of the modified mode $\mathrm{TM}_{\delta 10}(0<\delta<1)$ at frequency $2.56 \mathrm{GHz}$ is $1.83 \mathrm{~dB}$ (Figure $10(\mathrm{a})$ ), which is much lower than the conventional mode. So, this sort of dual band antenna can be a suitable choice where low gain and less directive dual band performance is a prior requirement.

4.3. Electric Field Distribution. From the electric field distribution at the plane $y=0$, it is apparent that for conventional $\mathrm{TM}_{110}$ mode electric field flips its sign passing from one side to the other side of the patch, thus satisfying the condition for broadside radiation (Figure 11(b)). But in case of modified $\mathrm{TM}_{\delta 10}(0<\delta<1)$ mode due to using metamaterial, phase change of electric field at the MNG-DPS interface occurs in such a way that the electric field flips from one side to the other side of the patch, as a result of which the electric field distribution of $\mathrm{TM}_{\delta 10}(0<\delta<1)$ mode looks like that of $\mathrm{TM}_{110}$ mode, thus also satisfying the condition for broadside radiation (Figure $11(\mathrm{a})$ ).

\section{Conclusions}

In the first section of this paper, a novel dual band circular patch antenna with high directivity performance in 


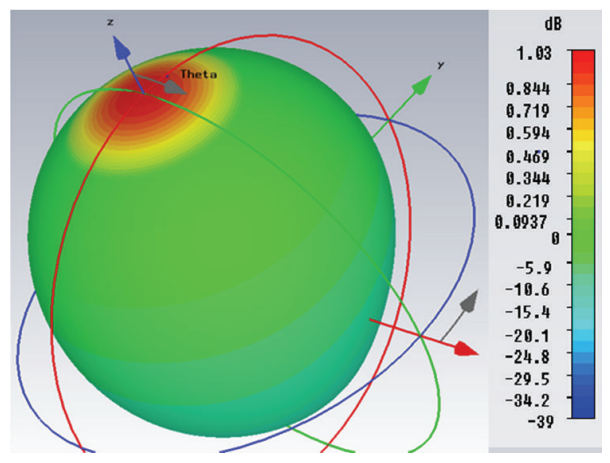

(a)

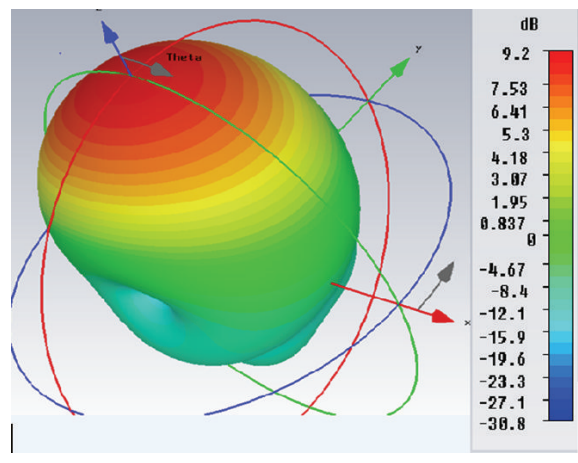

(b)

Figure 10: 3D view of (a) unconventional $\mathrm{TM}_{\delta 10}(0<\delta<1)$ mode's radiation pattern at $2.56 \mathrm{GHz}$ and (b) conventional TM $\mathrm{TM}_{110}$ mode's radiation pattern at $3.8 \mathrm{GHz}$.

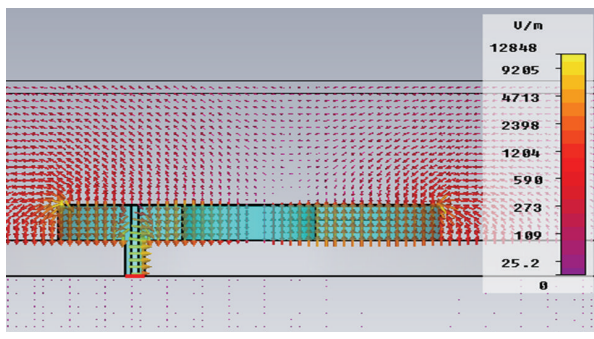

(a)

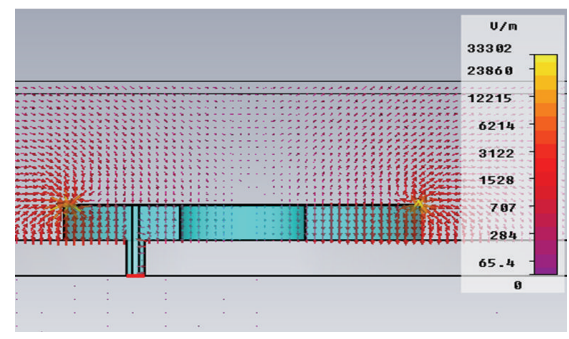

(b)

FIGURE 11: Electric field distribution on the $y=0$ plane on the metallic patch for the antenna of Figure 7 at (a) $2.56 \mathrm{GHz}$ for unconventional $\mathrm{TM}_{\delta 10}(0<\delta<1)$ mode and (b) $3.8 \mathrm{GHz}$ for conventional $\mathrm{TM}_{110}$ mode.

both bands has been shown. Inserting ENG metamaterial provides us with an additional modified mode with high gain. By controlling ENG metamaterial's filling ratio different frequency can be tuned in. So, this novel antenna design can be a suitable choice where highly directive gain along with user's flexibility in choosing resonant frequencies for dual band antenna purpose is required. In the second section of this paper, a miniaturized size dual band antenna loaded with MNG metamaterial has been designed. In this sort of antenna, since the unconventional mode yields a much lower gain than the conventional mode, one of its dual bands can be used as a substitute for low-gain antenna. Actually, this sort of antenna can be very effective where the use of less directive and low-gain small antennas is a prime concern. The use of this MNG loaded patch antenna is expected to be conducive for reasonably well signal propagation in some other special areas, such as high multipath interference regions, regions having frequent obstacles between transmitters and receivers, highly dense populated areas, and in spacecraft as a backup to the high-gain antenna.

\section{Conflict of Interests}

The authors declare that there is no conflict of interests regarding the publication of this paper.

\section{References}

[1] S. Ferdous, A. Hossain, S. M. H. Chowdhury, M. R. C. Mahdy, and M. Abdul, "Reduced and conventional size multiband circular patch antennas loaded with metamaterials," IET Microwaves, Antennas \& Propagation, vol. 7, no. 9, pp. 768-776, 2013.

[2] K.-L. Wong and G.-B. Hsieh, "Dual-frequency circular microstrip antenna with a pair of arc-shaped slots," Microwave and Optical Technology Letters, vol. 19, no. 6, pp. 410-412, 1998.

[3] A. Alù, F. Bilotti, N. Engheta, and L. Vegni, "Subwavelength, compact, resonant patch antennas loaded with metamaterials," IEEE Transactions on Antennas and Propagation, vol. 55, no. 1, pp. 13-25, 2007.

[4] K. S. Zheng, W. Y. Tam, and D. B. Ge, "Broadside subwavelength microstrip antennas partially loaded with metamaterials," in Proceedings of the International Workshop on Metamaterials (META '08), pp. 39-42, Nanjing, China, November 2008.

[5] F. Bilotti, A. Alu, and L. Vegni, "Design of miniaturized metamaterial patch antennas with $\mu$-negative loading," IEEE Transactions on Antennas and Propagation, vol. 56, no. 6, pp. 1640-1647, 2008.

[6] P. Y. Chen and A. Alù, "Sub-wavelength elliptical patch antenna loaded with $\mu$-negative metamaterials," IEEE Transactions on Antennas and Propagation, vol. 58, no. 9, pp. 2909-2919, 2010.

[7] J. Pruitt and D. Strickland, "Experimental exploration of metamaterial substrate design for an electrically small patch-like 
antenna," in Proceedings of the Antennas and Propagation Society International Symposium (APSURSI '10), IEEE, July 2010.

[8] S. Jahani, J. Rashed-Mohassel, and M. Shahabadi, "Miniaturization of circular patch antennas using MNG metamaterials," IEEE Antennas and Wireless Propagation Letters, vol. 9, pp. 11941196, 2010.

[9] J. Xiong, H. Li, B. Z. Wang, Y. Jin, and S. He, "Theoretical investigation of rectangular patch antenna miniaturization based on the DPS-ENG bi-layer super-slow TM wave," Progress in Electromagnetics Research, vol. 118, pp. 379-396, 2011.

[10] M. R. C. Mahdy, M. R. A. Zuboraj, A. A. N. Ovi, and M. A. Matin, "An idea of additional modified modes in rectangular patch antennas loaded with metamaterial," IEEE Antennas and Wireless Propagation Letters, vol. 10, pp. 869-872, 2011.

[11] M. Hassan, M. R. C. Mahdy, G. M. Hasan, and L. Akter, "A novelminiaturized triple-band antenna," in Proceedings of the 7th International Conference on Electrical and Computer Engineering (ICECE '12), pp. 702-705, Dhaka, Bangladesh, December 2012.

[12] CST Microwave Studio, CST of America, http://www.cst.com.

[13] S. M. H. Chowdhury, M. A. Hossain, M. S. Ferdous, M. R. Chowdhury Mahdy, and M. A. Matin, "Conceptual and practical realizationof reduced size multi-band circular microstrip patch antenna loaded with MNGmetamaterial," in Proceedings of the 7th International Conference on Electrical and Computer Engineering (ICECE '12), pp. 834-837, Dhaka, Bangladesh, December 2012. 

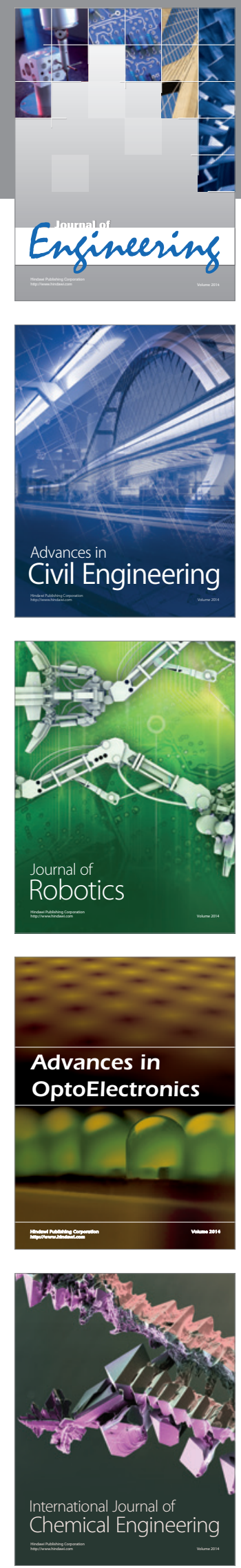

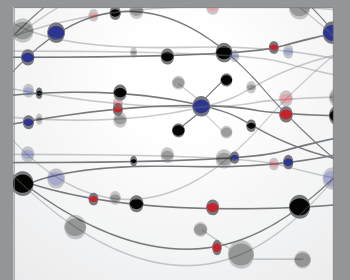

The Scientific World Journal
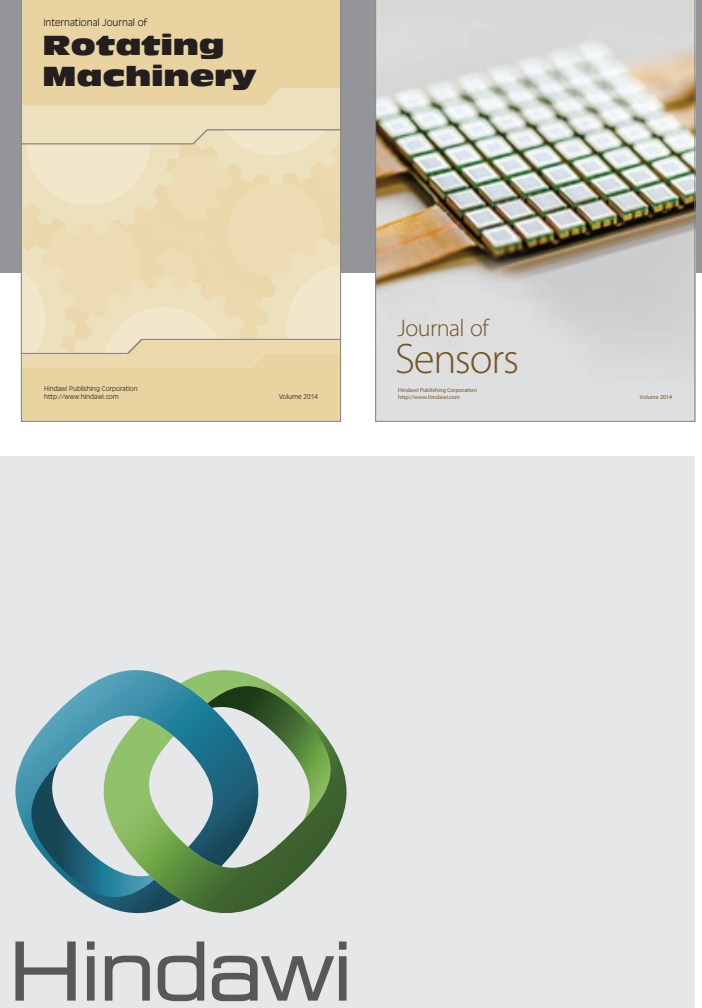

Submit your manuscripts at http://www.hindawi.com
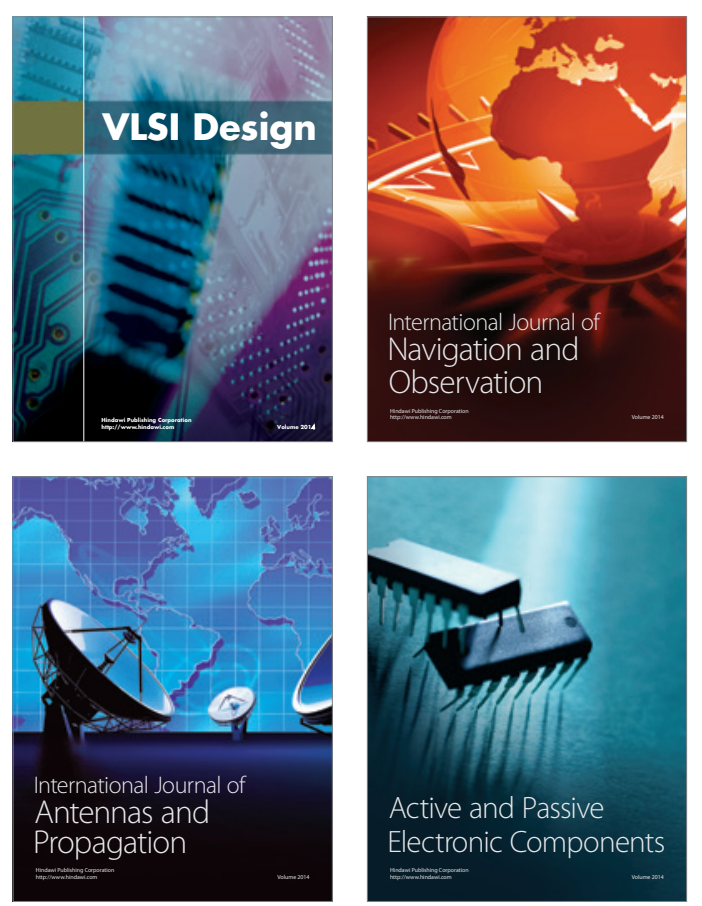
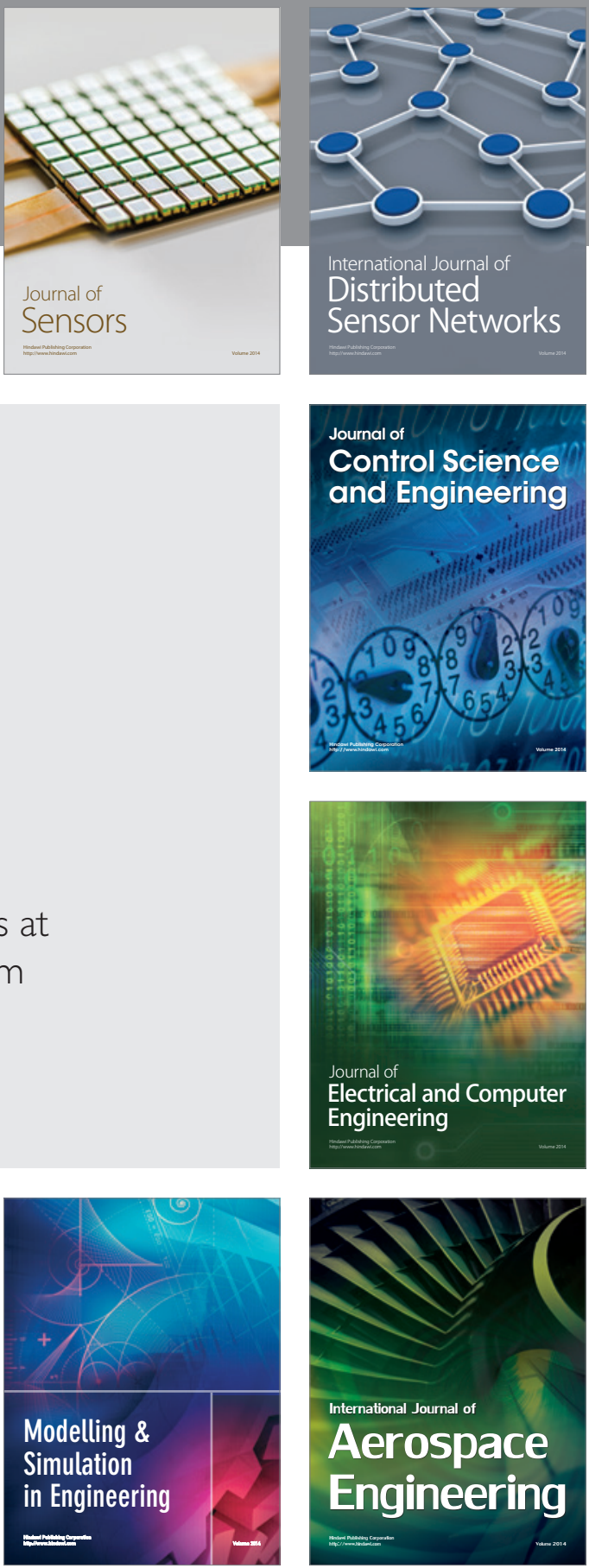

Journal of

Control Science

and Engineering
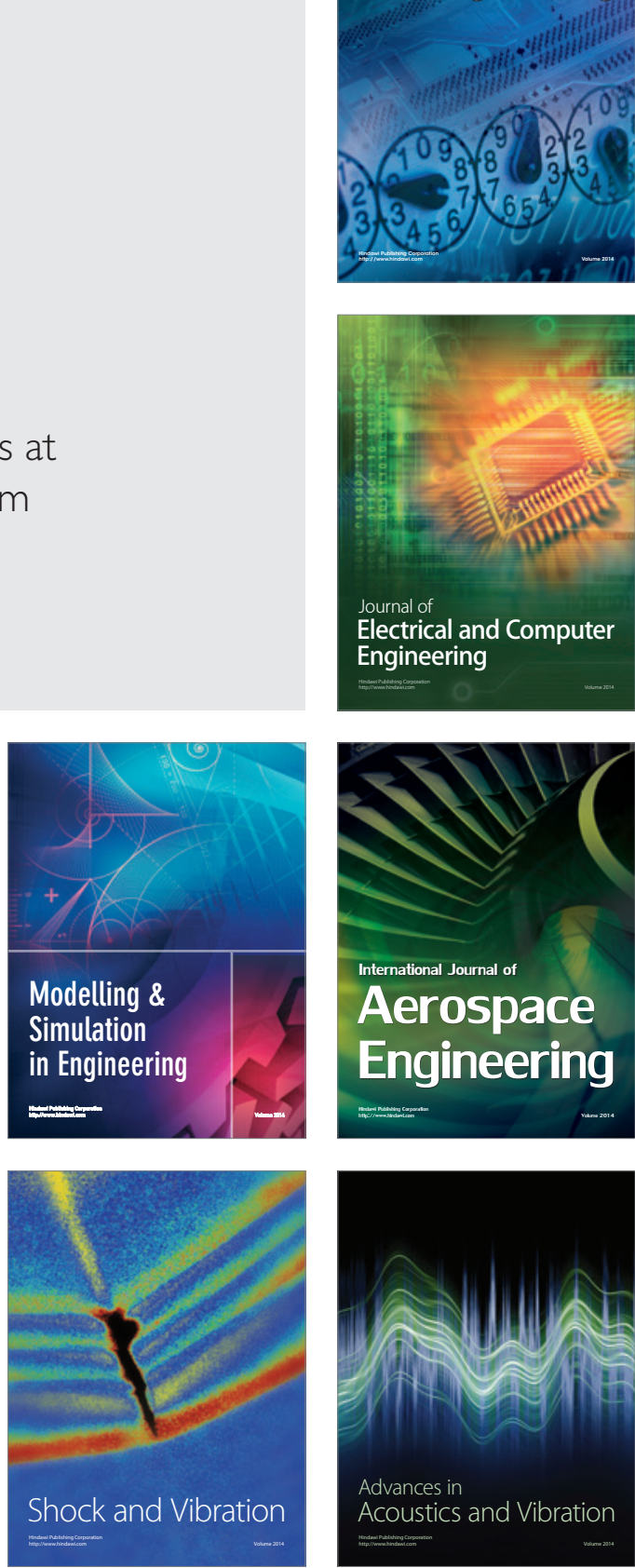